\title{
Facelock: Familiarity-based graphical authentication
}

Authentication codes such as passwords and PIN numbers are widely used to control access to resources. One major drawback of these codes is that they are difficult to remember. Account holders are often faced with a choice between forgetting a code, which can be inconvenient, or writing it down, which compromises security. In two studies, we test a new knowledge-based authentication method that does not impose memory load on the user. Psychological research on face recognition has revealed an important distinction between familiar and unfamiliar face perception: When a face is familiar to the observer, it can be identified across a wide range of images. However, when the face is unfamiliar, generalisation across images is poor. This contrast can be used as the basis for a personalised 'facelock', in which authentication succeeds or fails based on image-invariant recognition of faces that are familiar to the account holder. In Study 1, account holders authenticated easily by detecting familiar targets among other faces ( $97.5 \%$ success rate), even after a one-year delay ( $86.1 \%$ success rate). Zero-acquaintance attackers were reduced to guessing ( $<1 \%$ success rate). Even personal attackers who knew the account holder well were rarely able to authenticate (6.6\% success rate). In Study 2 , we found that shoulder-surfing attacks by strangers could be defeated by presenting different photos of the same target faces in observed and attacked grids (1.9\% success rate). Our findings suggest that the contrast between familiar and unfamiliar face recognition may be useful for developers of graphical authentication systems. 
Facelock: Familiarity-based graphical authentication

3

4

5

6
Rob Jenkins ${ }^{1 *}$, Jane L. McLachlan², \& Karen Renaud ${ }^{3}$

${ }^{1}$ Department of Psychology, University of York

2School of Psychology, University of Glasgow

${ }^{3}$ School of Computing Science, University of Glasgow

$7 *$ Correspondence to:

8 Dr Rob Jenkins

9 Department of Psychology

10 University of York

11 York

12 YO10 5DD

13 e:rob.jenkins@york.ac.uk 
14

15

16

17

18

19

20

21

22

23

24

25

26

27

28

29

30

31

32

33

34

35

36

37

38

39

40

41

42 t: +44 (0) 1904323144

Introduction

Security codes such as passwords and personal identity numbers (PINs) are widely used to control access to resources (e.g. bank accounts, websites, mobile devices). To protect against fraudulent access, it is essential that a security code should be difficult to guess (Garfinkel \& Spafford, 1996; Gehringer, 2002; Carstens \& Malone, 2009). From this standpoint, a random sequence of symbols (e.g. "8z3gxFtv") is a much better password than a user's own surname (e.g. "jenkins"). However, security codes that are difficult to guess tend also to be difficult to remember (Ebbinghaus, 1964; Craik \& Lockhart, 1972; Zviran \& Haga, 1990; Zviran \& Haga, 1993). For this reason, legitimate code holders are often faced with a choice between forgetting a code, which can be frustrating and inconvenient, or writing it down, which compromises security (Carstens, 2009; Tam, Glassman, \& Vandenwauver, 2010).

These and other weaknesses (Adams \& Sasse, 1999; Sasse, Brostoff, \& Weirich, 2001) have led developers to explore other forms of knowledge-based authentication, including graphical authentication (Blonder, 1996; Biddle, Chiasson, van Oorschot, 2012). In such systems, a user's authentication code is a set of images rather than an alphanumeric string. To log in, users identify their own images from larger challenge sets (Podd, Bunnell, \& Henderson, 1996; Brostoff \& Sasse, 2000; Dhamija \& Perrig, 2000; Furnell, Papadopoulos, \& Dowland, 2004; Weinshall \& Kirkpatrick, 2004). One of the most well developed of these systems is Passfaces (Brostoff \& Sasse, 2000), in which the images used are photographs of faces. Passfaces offers several advantages over standard passwords, most notably higher memorability of authentication codes (Paivio $\&$ Csapo, 1973). For example, an early evaluation found that after a 5-month delay, $72 \%$ of participants remembered their Passfaces codes on their first login attempt (Valentine, 1998). For comparison, a similar evaluation of passwords found that only $27 \%$ of passwords were remembered following a delay of 3 months (Zviran $\&$ 
43 Haga, 1993). In a pioneering field trial, Brostoff \& Sasse (2000) reported that login

44 failures were three times higher for passwords than for Passfaces. This estimate is

45 consistent with previous findings. However, such graphical systems are not

46 without their limitations (Furnell, Papadopoulos, \& Dowland, 2004; Tari, Ozok, \&

$47 \quad H o l d e n, 2006$; Everitt, Bragin, Fogarty, \& Kohno, 2009; Mihajlov \& Jerman-Blazic,

2011). Perhaps foremost among these is their susceptibility to 'shoulder-surfing'

attacks (Tari, Ozok, \& Holden, 2006), in which an attacker obtains a user's authentication code by secretly watching the user during authentication. This

$$
\text { attack is powerful because it exploits the memorability of image-based codes: }
$$

Images that are easy for the user to recognise are also easy for an attacker to recognise (Paivio \& Csapo, 1973).

In the present study we show that this symmetry - between ease of recognition for the user, and ease of recognition for the attacker - can be broken by applying insights from cognitive psychology research. Psychological studies of face recognition have revealed strong qualitative differences between processing of familiar and unfamiliar faces (Burton \& Jenkins, 2011; Jenkins \& Burton, 2011). When a face is familiar to the viewer, it can be identified from a wide range of different photographs, even when image quality is very poor (Harmon, 1973; Burton, Wilson, Cowan, \& Bruce, 1999; Burton, Jenkins, \& Schweinberger, 2011; see Figure 1). Importantly for this study, different images of a familiar face are almost never mistaken for different people (Jenkins, White, van Montfort, \& Burton, 2011). In contrast, our ability to identify unfamiliar faces from photographs is strikingly poor (Bruce et al., 1999, 2001). Very often, different photos of an unfamiliar face are seen as different individuals (Jenkins, White, van Montfort, \& Burton, 2011). Thus, familiarity with a particular face determines one's ability to identify it across changes in image (see Figure 2). Although the transformative effect of familiarity on face recognition may be not be intuitively obvious, it is highly robust, and has been replicated in dozens of experiments 

spanning decades of research (Bruce, 1982; Clutterbuck \& Johnston, 2002, 2004, 2005; Megreya \& Burton, 2006; Jenkins, White, van Montfort, \& Burton, 2011).

The familiarity contrast is normally encountered as a problem in applied settings. For example, unfamiliar face matching presents a serious challenge for security personnel and for automatic face recognition systems. In the present study we offer a very different perspective, by describing how the familiarity contrast might be exploited positively as the basis of an authentication system. The principle is straightforward: Familiarity with a particular face determines an observer's ability to identify it across different photographs. For any individual face that is not widely known, this ability will be very narrowly concentrated within the population. If a set of such faces is known only to a single individual, it can be used to create a personalized lock. Access is granted to anyone who demonstrates image-invariant recognition of the critical faces, that is, anyone who is familiar with them all. Conversely, access is denied to anyone who does not demonstrate image-invariant recognition of the critical faces, that is, anyone who is not familiar with them all.

To test this principle, we developed a prototype system that involves presenting a series of face arrays, similar to Passfaces. In our scheme, each array contains one face that is familiar to the user, among other faces that are unfamiliar. The user gains access by simply indicating the familiar face in each array. We refer to this method as Facelock. The scheme has two major advantages over traditional authentication methods. First, there is no explicit memory involved - the task is simply to pick out the familiar face in each array. As this task does not require the user to remember a code, the issue of forgetting one's code does not arise. Dispensing with a set code also means that the challenge arrays, and the familiar faces embedded in them, may be composed of different photographs of different individuals at each login. This is very different from the traditional approach of assigning a single invariant authentication code to an account holder. The second 
99 major advantage concerns the problem of writing down authentication codes

100 (Dunphy, Nicholson, \& Oliver, 2008; Tam, Glassman, \& Vandenwauver, 2010). In

101 the proposed scheme, the user is not required to reproduce a particular set code

102 in order to authenticate. The only requirement is to distinguish familiar faces from

103 unfamiliar faces. As familiarity discriminations are extremely robust (Young, Hay,

$104 \&$ Ellis, 1985; De Haan, Young, \& Newcombe, 1991), users have no incentive to

105 write down aide-memoires for their targets, and the associated security risk can

106 be avoided.

107 The main aims of the current work are i) to test the feasibility of an authentication

108 method that exploits the familiarity contrast in face recognition, and ii) to assess

109 its resilience against two very different forms of attack - guessing by high-

110 acquaintance attackers, and shoulder-surfing by zero-acquaintance attackers. The

111 aim at this stage is not to develop a commercially viable system. Instead we seek

112 to raise awareness of the important psychological contrast between familiar and

113 unfamiliar face processing, and to explore the potential for exploiting this contrast

114 in the context of authentication. We begin in Study 1 by comparing authentication

115 rates for legitimate account holders with authentication rates for i) zero-

116 acquaintance attackers, and ii) personal attackers who know the participants very

117 well (e.g. spouses, family members). In Study 2 we examine whether a full-

118 visibility shoulder-surfing attack can be thwarted by presenting different

119 photographs of the same targets to the participant and the attacker.

\section{Study 1}

121 The main aim of the first study was to establish whether participants could in

122 practice generate suitable target faces. These should be faces that the

123 participants know well, so that they could easily recognise them from

124 photographs, but that other people do not know well, so that all of the faces in the

125 array are unfamiliar to potential attackers. If such targets can be found, then it

126 should be possible to differentiate between account holders and attackers by 
127 comparing target detection performance. To anticipate, we found that suitable

128 targets were readily volunteered by participants. Authentication rates were very

129 high for legitimate users, even after a delay of one year. In contrast,

130 authentication rates for attackers were very low, even when the attackers were

$131 \quad$ close acquaintances of the users

$132 \quad$ Method

133

134

135

136

137

138

139

140

141

142

143

144

145

146

147

148

149

150

151

152

\section{Participants}

A total of 396 volunteers contributed data. 120 were volunteers who responded to our recruitment email (54 male, 66 female; age range 18-79). These 120

volunteers served as account holders in the current study. A further 110 volunteers were recruited from our participant pool to act as zero-acquaintance attackers, that is, people who knew nothing about the account holders. For comparison, we also asked each account holder to nominate two close acquaintances (e.g. spouses, family members) who could act as personal attackers. We reasoned that the faces of people who are familiar to participants might also be familiar to their close acquaintances, giving these personal attackers a significant advantage. We acknowledge that this personal attacker selection is unrealistic, as it assumes that attacks only ever come from close acquaintances, and never from strangers. However, we prefer here to underestimate the security of the system than to overestimate it. 166 nominated attackers took part. All account holders and nominated attackers were offered entry into a prize draw for an iPod Nano. The study received ethical approval from the FIMS Faculty Ethics Committee at the University of Glasgow (CSE 00871).

\section{Design and Procedure}

The study consisted of seven distinct phases - three preparation phases and four test phases. We describe each of these below. 
153

154

155

156

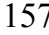

158

159

160

161

162

163

164

165

166

167

168

169

170

171

172

173

174

175

176

177

178

Phase 1: Target Nomination

Account holders nominated four or more target faces by entering the targets' names on the project website. As the proposed system relies on account holders and attackers having contrasting degrees of familiarity with the targets, appropriate selection of targets was critical. Ideally, an account holder's targets should be well known to the account holder, but unknown to other people. Our pilot work indicated that it can be difficult spontaneously to generate targets that satisfy both of these requirements. For this reason, we provided account holders with the following instructions in order to guide them to the appropriate region of their search space. Figure 3 represents the constraints on target selection schematically.

"The next page will ask you to list some minor celebrities - really minor celebrities.

Almost everyone recognises the 'A-List' celebrities below [photos of international celebrities such as major film stars]. Most people also recognise some 'B-List' celebrities [photos of national celebrities such as television presenters]. We want you to tell us your 'Z-List' celebrities.

By 'Z-List' celebrities, we mean people who are (or were):

1. Only famous within a narrow field of interest. For example, a famous skier or a famous cellist. This could include someone who was famous many years ago, but who is not well known these days.

2. Well known to you, so that you would easily recognise them from photographs.

3 Not well known to the public at large, so that you would not expect others to recognise them. 
180

181

182

183

184

185

186

187

188

189

190

191

192

193

194

195

196

197

198

199

200

201

202

203

204

205

206

Having read these instructions, account holders were asked to submit the names of four or more targets, up to a maximum of ten. There was no time limit for this task, and account holders were free to log out and return later to complete it. Once an account holder was satisfied with this personal list, the names were transferred to the experimenter. Each account holder was also asked to provide email addresses of two close acquaintances (e.g. spouses, family members) who would be willing to act as personal attackers.

\section{Phase 2: Image Collection}

Targets who had already been nominated by another account holder $(<1 \%)$ were eliminated to avoid ambiguity at login. For all other targets, the experimenter collected at least four face photographs by using the target's name as a Google Image search term. We accepted the first four photographs in which the whole face was visible, regardless of viewing angle, lighting, age, or other sources of image variability. This resulted in 4 different photographs for each of 603 faces (2412 images in total). All photos were cropped to a rectangular frame measuring 100 pixels wide $\times 119$ pixels high for presentation. The collected photos of each account holder's targets were then uploaded to the project website for that account holder to approve.

Phase 3: Image Approval

Account holders returned to the website to view the photos of their targets and to approve or decline each image. The purpose of this step was twofold. First, it allowed us to ensure that the photos depicted the correct individual. This was necessary as names are rarely unique identifiers, and search results invariably included images of more than one person. Second, it allowed us to confirm that the returned images were indeed recognisable to their nominators. Declined images $(<1 \%)$ were replaced until the account holder was satisfied with the selection. Image approval was followed by a delay of one week to allow forgetting of the selection 
207 procedure. Account holders then received an email requesting them to return to

208 identify their faces again.

209 Phase 4: Account Holder Login (one week delay)

210 After the one-week delay, account holders returned to the project website and

211 attempted to authenticate. The account holder's lock consisted of a series of four

212 different challenge sets, each comprising nine face photographs arranged in a $3 \times 3$

213 grid (similar to Passfaces challenge sets; see Figure 4). In each grid, one image (the

214 target) was a random photo of a person selected at random from that account

215 holder's pool of target names. The remaining eight images (the distractors) were

216 random photos of faces drawn at random from other account holders' pools of

217 targets. Allocation of the nine images to the nine grid positions was randomised so

218 that location was not predictive of target/distractor status. This meant that from the

219 perspective of the account holder, each grid contained one familiar face among

220 eight unfamiliar faces. However, from the perspective of an attacker, all nine faces

221 should be unfamiliar. The account holder's task was simply to click on the familiar

222 face in each grid. Identifying the correct image in all four grids resulted in successful

223 authentication. The probability of opening the lock by chance alone was thus 1 in

224 6561, or $0.015 \%$, for this particular instantiation.

225 No feedback was given until the end of the four-grid lock, after which the account

226 holder was told whether or not the authentication attempt was successful. If the

227 attempt was unsuccessful, the lock was reset using newly selected photos, and the

228 account holder was asked to try again. Following successful authentication, or three

229 unsuccessful attempts, the account holder proceeded to a brief questionnaire

230 concerning account holders' impressions of the system.

231 Phase 5: Zero-Acquaintance Attacker Entry

232 In small-scale pilot studies, we found that medium-acquaintance attackers (work

233 colleagues) were never successful. To estimate the success rate in a larger sample,

234 we recruited 114 zero-acquaintance volunteers to attack a randomly allocated lock. 
235 These 114 volunteers undertook 207 attacks between them. The authentication

236 procedure for the attacker phase was exactly the same as for the account holder

237 phase, with one of the account holder's targets and eight non-target faces making

238 up each grid. As with the account holder entry phase, no performance feedback was

239 given until successful authentication, or three unsuccessful attempts. We expected

240 that if the account holder chose appropriate targets, none of these faces should be

241 familiar to the attacker, and the success rate should not exceed chance levels. The

242 zero-acquaintance attackers were recruited to verify that this was the case.

243 However, our main interest was in the success rate of the personal attackers.

244 Phase 6: Personal Attacker Entry

245 In the first phase of the study, each account holder was asked to provide email

246 addresses of two close acquaintances who would be willing to act as personal

247 attackers. A total of 166 personal attackers agreed to take part, undertaking 249

248 attacks between them. Importantly, attackers only attacked their own nominator, so

249 that every attack was from a close personal acquaintance of the account holder (e.g.

250 spouse, family member), rather than from a stranger. Again, the authentication

251 procedure was the same as for the account holder phase. If the account holder

252 chose appropriate targets, all of the faces in all of the grids should be unknown to

253 the attacker. We reasoned that high-acquaintance attackers might have acquired a

254 degree of familiarity with their nominators' targets, due to shared exposure (e.g.

255 overlapping interests or media consumption), thus providing a more stringent test.

256 As with the account holder entry phase, no performance feedback was given until

257 the end of the entire four-grid sequence that comprised a single lock. Following

258 successful authentication, or three unsuccessful attempts, the attacker proceeded to

259 a brief questionnaire concerning attackers' impressions of the system.

260 Phase 7: Account Holder Login (One year delay)

261 One year after the initial account holder login phase, account holders were asked to

262 authenticate a second time. This was the only contact between experimenters and 
263 account holders since the initial login phase, and our log confirmed that none of the

264 participants had visited the project website during the intervening months. Thus, the 265 one year interval provided an excellent opportunity for account holders to forget

266 about the study (Ebbinghaus, 1964). Previous research has shown that passwords

267 are quickly forgotten once they fall into disuse (Witty \& Brittain, 2004). For example,

268 two studies of password memorability (Zviran \& Haga, 1990, 1993) reported

269 memorability rates of $35 \%$ and $27.2 \%$ after a delay of five months. Given that a

270 putative advantage of our familiarity-based approach is that it imposes no memory

271 load, we predicted relatively preserved authentication rates even after a year of

272 disuse.

\section{Results and Discussion}

274 Authentication data

275 As can be seen in Table 1,97.5\% of account holders (117/120) successfully

276 authenticated, with 84.2\% (101/120) succeeding on the first attempt. In contrast,

277 only $6.6 \%$ of personal attackers (11/166) were successful, and only 3.0\% (5/166)

278 on the first attempt. This compares favourably with previous analyses based on

279 Passfaces (Davis, Monrose, \& Reiter, 2004).

280 Chi Square analysis of these total success rates confirmed a highly significant 281 difference between account holders and personal attackers $\left[\chi^{2}(1)=232.6, p<\right.$.

282 0001]. We also note that the majority of account holders' failures to authenticate 283 were 'near misses', in which three of the four targets were correctly identified. For 284 personal attackers, near misses were the least frequent authentication failure.

285 Only one attack by a zero-acquaintance attacker was successful, precluding any 286 statistical analysis for this group. However, the circumstances of the one 287 successful attack are perhaps revealing. Specifcally, the account holder had not 288 chosen 'Z-List' celebrities as required. Indeed, for the successfully attacked lock, 
289 two of the four faces were members of the rock band Led Zeppelin (Robert Plant,

290 Jimmy Page), perhaps analogous to choosing "ledzeppelin" as a password.

291 Analysis of the 11 successful attacks by nominated attackers revealed similar 292 regularities. In five of these cases, the account holders had chosen widely-known 293 celebrities as targets (e.g. Tony Blair, John Wayne), instead of 'Z-List' celebrities. 294 In a further three cases, the account holders were non-Caucasian, and chose only 295 non-caucasian target faces. Since virtually all of the distractor faces were 296 Caucasian, these account holders' targets were presumably easy for their 297 nominated attackers to guess. Nominated attackers were always close 298 acquaintances of the account holders in this study, and so knew the ethnic 299 background of the account holders they were attacking. For the remaining three 300 successful attacks, we suggest that the attackers had some degree of familiarity 301 with their account holders nominated targets - enough to set the targets apart 302 from the distractors. For example, musicians that one likes might be recognized 303 by one's spouse, due to shared exposure.

304 Taken together, the success rates of account holders (97.5\%), randomly zero305 acquaintance attackers $(<1 \%)$, and nominated high-acquaintance attackers $306 \quad(6.6 \%)$ strike us as a promising starting point. Analysis of successful attacks 307 provides little evidence that the principle of exploiting familiarity contrast is 308 problematic. Rather, the main challenge is the separable problem of compliance: 309 If the system is not used as intended, it does not work as well. This limitation is 310 characteristic of a wide range of security systems - including passwords, PIN $311 \quad$ codes, and mechanical locks. 
31379 of our initial account holders returned to login a second time, following a one-

314 year delay. As can be seen in Table 1, 86.1\% of these returning account holders 315 (68/79) successfully authenticated, 78.5\% (62/79) on their first attempt. This is a 316 remarkably well-preserved success rate over such a long period of disuse, especially 317 given that different images of the account holders' targets were presented at the 318 delayed login. For comparison, previous research reported a first-attempt 319 authentication rate of $77 \%$ after only two weeks when using tra ditional passwords 320 (Bunnell et al., 1997). Established graphical authentication systems are also 321 vulnerable to memory decay, though generally to a lesser degree than passwords. 322 One influential study (Valentine, 1998) reported an authentication rate of 72\% (by 323 third attempt) after a five-month delay when using Passfaces. Although these 324 comparisons involve rather different authentication methods, they highlight the very 325 different demands of recall-based, recognition-based, and familiarity-based 326 decisions.

327 328

We attribute account holders' high success rate in the present study to two main factors. First, there was no authentication code to remember, so the classic problem of account holders forgetting authentication codes did not apply. Second, our account holders had already established robust mental representations of their target faces prior to the study (they were familiar faces), so presenting different images of these targets did little to impede recognition (Jenkins \& Burton, 2011). Interestingly, a number of returning account holders commented on the surprising ease of authentication under these conditions. One wrote, "I didn't think I could log in because I couldn't remember any of the people I chose - but I did!" Interestingly, another reported, "I got them all right. Did you use the same images of the people or different ones? I got the impression that I did not recognise the image but the person."

\section{Account Holders' Questionnaire Data}


$340 \quad$ Account holders responded to five questionnaire items concerning user

341 experience. Summaries of these responses can be seen in Table 2.

342 The questionnaire data contain little evidence that account holders had difficulty

343 using this system. None of the account holders reported writing down their

344 targets' names. This suggests that they correctly understood that forgetting their

345 targets was not an issue. Only 10\% of account holders reported difficulty in

346 identifying their target faces. Thus most account holders were successful in

347 nominating faces that they could recognise well. Interestingly, the great majority

348 of account holders (80\%) stated that with the benefit of hindsight, they would

349 have chosen different targets. Presumably, since account holders had little trouble

350 recognising targets that they actually chose, their motive here was not making

351 authentication easier for themselves, but making it harder for attackers. 16\% of

352 account holders reported recognising one of the non-target faces in a grid.

353 However, the overall authentication rate of $97.5 \%$ implies that this confusion

354 rarely stopped them from authenticating correctly. On the basis of this

355 experimental trial, 31\% of participants said that they would use a Facelock system

356 instead of a password, 25\% said they would not, and 44\% were undecided. Given

357 that we made no concessions to usability and $\mathrm{HCl}$ issues in this study, it is

358 perhaps surprising that $31 \%$ of respondents were positively disposed to the

359 method.

\section{Personal attackers' Questionnaire Data}

361 Personal attackers responded to four questionnaire items using a 5-point Likert 362 scale, where 1 indicates a low rating, and 5 indicates a high rating. Mean ratings 363 for each item are shown in Table 3.

364 Personal attackers found guessing their account holders' targets moderately

365 effortful, and found it quite difficult to imagine who the account holder might have 366 chosen. Consistent with these impressions, they rated their level of success as 367 rather poor overall, though even this rating is a generous appraisal of their actual 
368 success rate. Personal attackers knew their account holders very well overall, confirming good compliance among account holders at the attacker nomination stage. To test whether personal attackers were more successful the better they knew their victims, we computed the correlation between these attackers' acquaintance ratings for Item 4 above, and the number of correctly-guessed targets (0-4) in their first attacks (see Figure 5).

This correlation was moderately positive and highly reliable $[R=0.29, N=166, p$ $<0.001$ ]. Importantly, lower acquaintance attackers (ratings <4) were never successful. We return to the issue of acquaintance in the General Discussion section.

\section{Study 2}

The preceding study confirmed that account holders who were familiar with the target faces could easily distinguish these faces from unfamiliar non-targets, regardless of the particular photos that were used to portray them. In contrast, attackers found it very difficult to guess account holders' targets, even when the attackers were close acquaintances of the account holders.

The second study focuses on a different aspect of the proposal, specifically the use of multiple photos of each target. We also sought to compare the resilience of different account holders' locks directly, by exposing them to multiple attacks. To this end, we modelled a best-case scenario for shoulder-surfing attacks, in which we presented the correct authentication sequence to attackers under full-visibility viewing conditions, and then asked them immediately to replicate the sequence using different photographs of the same target faces. Attackers were thus required to generate the sequence of identities that they had just seen, even though those identities were portrayed using different images.

As in Study 1, we loaded this situation heavily in the attackers' favour. First, we used the same four target identities for the observation sequence and the replication sequence, rather than drawing a set of four targets at random from the account 
396 holders' entire pool. Second, we presented these same four targets in the same

397 order in both sequences, rather than presenting them in a different random order

398 each time. Third, attackers did not have to glance furtively at the authentication

399 sequences for fear of being noticed. Instead, we presented the sequences very

400 clearly to the attackers, who were asked to give it their full attention. Finally, there

401 was no delay between the observation sequence and the replication task. Thus

402 attackers' memory decay was minimized. These real world complications were

403 eliminated in an effort to isolate the impact of a photo change. It is already

404 established that replicating a four-item sequence is well within the limits of human

405 short-term memory. This is true in experimental settings (Miller, 1956), and also in

406 the context of shoulder-surfing 4-digit PIN numbers (Anderson, 1993). However, the

407 present case differs from previous studies in that different images of each item are

408 used at the sequence replication stage. If attackers are able to integrate across

409 different photos of each target efficiently, then performance should be close to

410 ceiling (Miller, 1956). Alternatively, if a change in photograph impedes identification

411 in this situation, then performance should be relatively poor, even when the

412 authentication code is clearly presented to the attacker immediately before the

413 attack.

414 Method

415 Participants

416 Thirty-two postgraduate volunteers (6 male, 26 female; age range 21-36) completed

417 the study. The study received ethical approval from the FIMS Ethics Committee at

418 the University of Glasgow.

419 Design and Procedure

420 Each participant attacked five locks so that each lock was attacked 32 times. The

421 five locks (i.e. 5 different 4-grid sequences) were drawn at random from those that

422 led to successful authentication by account holders in Study 1 . In other words, the 
423 authentication rate for account holders was 100\% for this sample of locks. For each

424 lock, a different-image version was also constructed, by replacing the target from

425 each grid with a different photo of the same person, and replacing the eight non-

426 targets with different non-targets.

427 As with the original grids, the location of the images in the grid was randomised. To 428 make the task as easy as possible for the attackers, grid order was preserved across

429 observation and replication sequences, so that the same targets appeared in the 430 same order (1-4) in both versions of the lock. The different-image versions of the 431 grids were printed at a size of $10 \mathrm{~cm} \times 12 \mathrm{~cm}$ and bound into response booklets. The 432 original grids were projected at a size of $150 \mathrm{~cm} \times 180 \mathrm{~cm}$ using a computer 433 controlled data projector, which attackers viewed at a distance of between 3 and 5 434 metres.

435 For each of the five locks, attackers first watched the authentication sequence using 436 the original grids, and then tried to replicate the sequence on the different-image 437 grids, that is, to copy the account holder's authentication code. To demonstrate each 438 sequence as clearly as possible, each one of the four grids was presented on screen 439 for 5 seconds together with its grid number (1-4). After the first 2 seconds, a green 440 frame appeared around one of the faces, identifying that face as the target 441 (analogous to watching the account holder select that face). As face identification is 442 normally accomplished within about 200 msec of stimulus onset (Liu, Harris, \& 443 Kanwisher, 2002), we expected this presentation time to allow full encoding of the 444 correct target. This procedure was intended to model observation of target selection 445 in an optimal shoulder surfing situation, in which all the necessary information is 446 presented clearly at the focus of attention. Readers are invited to simulate this task 447 for a single grid by comparing Figure 6 and Figure 4.

448 Successive grids in each lock were separated by a blank interval of 2 seconds.

449 Immediately after the fourth target had been revealed, attackers were asked to 450 reproduce the sequence they had just seen, by circling the same four targets on 
451 their response sheets. There was no time limit for this task. When the attackers were

452 ready to proceed ( $<60$ secs in all cases), the next authentication sequence was

453 initiated. All 32 participants attacked the same 5 locks once, resulting in 160 attacks

454 in total.

\section{Results and Discussion}

456 Raw frequency data are shown in Table 4. Only 3 out of 160 attacks were successful

457 (1.9\%). This strikes us as a very promising figure, especially given the privileged

conditions of attack. When attempting to replicate the authentication sequence,

459 attackers saw the same targets presented in the same order under highly favourable 460 viewing conditions and with no time pressure. Only the photo used for each face was changed. As it turned out, this alone was enough to defeat these shoulder-surfing 462 attacks.

We note that all three successful attacks were on the same lock. Inspection of the 464 targets in this particular lock suggests that this may be due to their distinctive 465 appearance. For example, one of the targets was bald and wore glasses in both photos; another was an elderly woman with permed white hair. As none of the 467 distractor faces shared these features, the matching targets were presumably rather 468 salient in this context. In the General Discussion we consider how this situation could be avoided

\section{General Discussion}

\section{Summary of Findings}

472 Two studies tested a knowledge-based authentication system that exploits the 473 psychological contrast between familiar and unfamiliar face recognition. In Study 1

474 we found that account holders were able to generate target faces that were well 475 known to themselves, but were not well known to other people. Account holders 476 authenticated easily by detecting these familiar targets among other faces (97.5\% 477 success rate), and this was the case even after a one-year delay (86.1\% success 
478 rate). By contrast, zero-acquaintance attackers were reduced to guessing ( $<1 \%$

479 attacks rate). Even personal attackers who knew the account holder well were rarely

480 able to authenticate (6.6\% success rate). This success rate for attacks compares

481 favourably with previous studies. Analysing a system based on Passfaces, Davis et al

482 (2004) conclude that 10\% of authentication codes could be guessed within one or

483 two attempts, even by very low acquaintance attackers who know only the gender

484 or race of the account holder. Here we found a successful attack rate of $6.6 \%$ within

485 three attempts for very high acquaintance attackers who knew a great deal about

486 the account holder. In Study 2 we found that optimal shoulder-surfing attacks by

487 strangers could be repelled simply by using different photos of the targets in the

488 observed and attacked grids (1.9\% success rate). Together, these findings suggest

489 that the contrast between familiar and unfamiliar face recognition may be useful for

490 graphical authentication systems. Although face-based systems have been

491 developed previously, these have always conflated face recognition and image

492 recognition, by representing each face with a single image (Jenkins, White, van

493 Montfort, \& Burton, 2011). As image memory will be equally excellent for account

494 holders and attackers, such systems are vulnerable to shoulder-surfing attacks (Tari,

495 Ozok, \& Holden, 2006). The use of different photographs for each target confounds

496 attackers who are unfamiliar with the targets, but does not impede legitimate users

497 who are familiar with their chosen targets.

498 The approach we describe here offers two advantages. First, unlike a conventional

499 password, it does not require the account holder to remember anything specific to

500 the authentication procedure, as the task is simply to indicate which of several faces

501 is familiar. The system thus exerts very little memory load compared with

502 conventional passwords. Our most striking evidence for this comes from the delayed

503 authentication task in Study 1. Here, account holder's authentication rate was 86\%,

504 one year after a single login. This is unprecedented for knowledge based

505 authentication systems (Sasse, Brostoff, \& Weinrich, 2001). For comparison, one

506 evaluation of traditional passwords reported authentication rates of $27 \%$ after just 3 
507 months (Zviran \& Haga, 1993). A similar evaluation of Passfaces found

508 authentication rates of $72 \%$ after 5 months (Valentine, 1998). Such studies

509 contribute to the general finding that memory decay impacts image recognition less

510 than it impacts password recall. Here we show that memory decay impacts face

511 familiarity judgements even less. Second, it does not matter greatly if authentication

512 is observed. As Study 2 shows, even when an attacker sees the same set of targets

513 when attempting to authenticate, authentication is still difficult when different

514 photos of those targets are presented. Previous work has shown that Passfaces is

515 highly vulnerable to shoulder surfing when a mouse pointer is used to select targets.

516 Participants in that study rated the vulnerability of Passfaces at 5.2 on a scale from 1

517 (not vulnerable) to 7 (extremely vulnerable), indicating that shoulder surfers found it

518 very easy to obtain the faces by observation. In the same study, dictionary based

519 passwords were rated 4.85 in terms of vulnerability. Interestingly, using a keyboard

520 instead of a mouse to select targets reduced the vulnerability of Passfaces from 5.2

521 to 2.3, presumably because keyboard entry forced onlookers to divide their attention

522 between the screen and the keyboard (Braun, 1998). For the same reason, keyboard

523 input should strengthen the scheme we propose here.

\section{Limitations}

525 Our testing exposed a number of important limitations to the system in its

526 experimental form. First, the lock is vulnerable to an attacker who, like the account

527 holder, knows the target faces. This was evident in Study 1, in which attackers who

528 were closest acquaintances of the account holders correctly guessed more targets

529 than attackers who were less close acquaintances. This vulnerability underscores

530 the importance of appropriate target selection. One way for a secret holder to

531 minimise risk would be to maintain a large pool of target faces, and to sample these

532 from disparate fields of interest, so that no single attacker knows enough targets to

533 authenticate. 
534 A second limitation is that attackers may be able to match different images of

535 targets whose appearance is both distinctive (e.g. bald head and round glasses), and

536 stable (i.e. similar appearance in all photos). This was seen in Study 2, where one

537 lock that contained highly distinctive faces could be compromised in a shoulder-

538 surfing attack. For similar reasons, target distinctiveness may be a concern

539 whenever an account holder's targets are all drawn from a single ethnic group or

540 age band. These risks could be reduced by avoiding highly distinctive faces, and by

541 avoiding similar images of any particular target.

542 De Angeli et al. (2005) proposed that graphical authentication mechanisms such as

543 Facelock should be assessed in terms of guessability, observability and recordability 544 when considering how they can be breached. Table 5 shows a threat model based on 545 this taxonomy.

\section{$546 \quad$ Future Directions}

547 One pragmatic concern is scalability. Our experimental implementation of Facelock 548 involved a multi-step enrollment process, and required considerable human labour 549 to find images of targets and verify these with the account holders. This may not be 550 feasible for a large-scale system. Unless these steps can be significantly

551 streamlined, the approach may be better suited to small-scale or personal 552 deployments such as locking computers and mobile devices than to large-scale 553 deployments such as securing bank accounts.

554 The studies we report here suggest a number of possible directions for future 555 development. One would be to select non-targets automatically for each grid based 556 on their similarity to the target. For example, if the target for a particular grid is a 557 young Asian female, the non-targets used to complete that grid could also be young 558 Asian females. Increasing the homogeneity of the grids should undermine attacks 559 that rely on distinctiveness to infer targets (Study 1). This functionality would require 
560 all images in the system to be tagged with properties such as age, sex, and race.

561 Automatic tagging is currently a major focus of image analysis (Datta, Joshi, Li, \&

562 Wong, 2008), and much progress has been made in recent years (see Bengio, 2009,

563 for an instructive overview). Indeed, human similarity ratings of faces can already be

564 accurately predicted by automatic systems (Lacroix, Postma, \& Murre, 2005), which

565 could dramatically improve the effectiveness of facelock image arrays.

566 We noted in Study 1 that $80 \%$ of account holders would choose different targets if

567 they could choose again. As authentication failures were so rare among these

568 account holders, it seems reasonable to assume that they would not have chosen

569 different targets to make their own authentication even easier, but rather to make

570 fraudulent access even harder. Presumably faces that are less widely known

571 occurred to these account holders after the study had begun, and the account

572 holders realised that these would make better targets. If so, allowing account

573 holders to update their pool of target faces could improve the security of the system.

574 A related issue concerns the optimal number and set size of the grids that are used

575 to authenticate. In the present studies we arbitrarily chose a sequence of four $3 \times 3$

576 grids, which corresponds to a guessing rate of 1 in 6,561. It would be technically

577 trivial to change the guessing rate by changing the grid configuration (e.g. 1 in

$5781,048,576$ for 5 different $4 \times 4$ grids), but implementation details are not our priority

579 here. Our main concern is whether familiarity contrasts in face recognition may be

580 exploited to improve the security of authentication systems. This question is

581 independent of any particular grid configuration. Dedicated usability studies will be

582 required to examine trade offs between security and ease of use. Such studies

583 should also seek to optimise task instructions to make them as easy as possible to

584 follow. In Study 1, five of the eleven successful attacks from personal attackers, and

585 the single successful attack from a random attacker, were all attributable to account

586 holders nominating major celebrities as their targets, despite instructions to the

587 contrary. Clearer instructions, or tighter constraints on the target nomination

588 process, could mitigate this vulnerability. 
589

590

591

592

593

594

595

596

597

598

599

600

601

602

603

604

605

606

607

608

609

610

611

612

613

614

615

616

\section{Concluding remarks}

Although we have outlined a novel approach to graphical authentication using faces, there are clearly very many issues outstanding. In this final section we highlight some of these in the hope that we can be as clear as possible in articulating what is and is not claimed for this proposal.

- We are not presenting Facelock as a packaged product that is ready to deploy. Instead we offer these initial studies as proof of principle. Our focus throughout is on the familiarity of a face to the observer, and how this profoundly affects the observer's ability to process images of that face. The key contrast between familiar and unfamiliar face perception has seldom been addressed in the computer science literature (Sinha, Balas, Ostrovsky, \& Russell, 2006). Here we hope to have demonstrated that this contrast may be usefully exploited in graphical authentication systems. However, a number of usability issues (discussed above) would need to be resolved before such a system could be practically deployed.

- We do not claim that the proposed system is flawless. In the studies we present, some account holders failed to authenticate, and some attackers succeeded. We address both of these outcomes, alongside other limitations of the studies, in the discussion section of the paper. Our main emphasis is the relative performance of observers who are familiar or unfamiliar with the faces concerned. In perceptual experiments, recognition performance is radically different for these two groups. Here we show that the same applies when the task is incorporated in an authentication system.

- We are not claiming that Facelock is superior to Passfaces. Any such evaluation would require a direct comparison of the two approaches, and we have not attempted that here. Previous studies have looked at memorability of Passfaces (Valentine, 1998) and its susceptibility to shoulder-surfing attacks (Tari, Ozok, \& Holden, 2006), and we consider these issues also. However, 
617

618

619

620

621

622

623

624

625

626

627

628

629

630

631
Passfaces is an established commercial system. Facelock, as an experimental proposal, is unfettered by implementation concerns. Any attempt to compare performance directly would thus be rather unfair on Passfaces. Indeed, the general question of which system authentication system is 'best' is likely too simplistic. Any approach will have its own profile of strengths of weaknesses, and will be better suited to some situations - and to some users - than to others.

- We do claim that it is easy for users to generate a set of faces that are well known to them, but not to other people. We show that an authentication code based on such faces makes it easy for the user to login, even after a year of disuse, as it does not require the user to commit anything to memory. The user's authentication code is difficult for other people to guess, even for close acquaintances such as spouses. It is also highly resistant to shoulder-surfing, as image changes that are transparent for the (familiar) user are not transparent for the (unfamiliar) attacker. 
632 More generally, we propose that research into graphical authentication systems can

633 exploit findings from psychological research, and that psychological research can be 634 enriched by considering applied problems in other fields. Image recognition is not

635 the same as face recognition. Unfamiliar face recognition is not the same as familiar

636 face recognition. Not all observers are equal. These insights offer much scope for 637 innovation in face-based graphical authentication systems, and we hope that the 638 current studies might spur further development in this direction. References

639 Adams, A., \& Sasse, M.A. (1999). Users are not the enemy: Why users compromise 640 computer security mechanisms and how to take remedial measures.

641 Communications of the ACM, 42, 41-46.

642 Anderson, R (1993). Why cryptosystems fail. Proceedings of the 1st ACM conference 643 on Computer and communications security, 215-227.

644 Bengio, Y. (2009). Learning deep architectures for Al. Foundations and Trends in Machine 645 Learning, 2, 1-127.

646 Biddle, R., Chiasson, S., \& Van Oorschot, P. C. (2012). Graphical passwords: Leaming from the first 647 twelve years. ACM Computing Surveys, 44, 1-19.

648 Blonder, G. (1996). Graphical passwords. US Patent 5559961.

649 Braun, J. (1998). Divided Attention: Narrowing the gap between brain and behavior. In R. Parasuraman, 650 editor, The Attentive Brain, pages 327-351. MIT Press, Cambridge, Massachusetts.

651 Brostoff, S., \& Sasse, M. A. (2000). Are Passfaces more usable than passwords? A field trial investigation, 652 in: S. McDonald, Y. Waem \& G. Cockton [Eds.]: People and Computers XIV - Usability or Else! 653 Proceedings of $\mathrm{HCl} 2000,405-424$.

654 Bruce, V. (1982). Changing faces. Visual and non-visual coding processing in face recognition. British 655 Journal of Psychology, 73, 105-116. 
656 Bruce, V., Henderson, Z., Greenwood, K., Hancock, P., Burton, A. M. \& Miller, P. (1999). Verification of 657 face identities from images captured on video. Journal of Experimental Psychology: Applied, 5, 339658360.

659 Bruce, V., Henderson, Z., Newman, C., \& Burton, A. M. (2001). Matching identities of familiar and 660 unfamiliar faces caught on CCTV images. Journal of Experimental Psychology: Applied, 7, 207-218.

661 Bunnell, J., Podd, J., Henderson, R., Napier, R., \& Kennedy-Moffat, J. (1997). Cognitive, associative and 662 conventional passwords: Recall and guessing rates. Computers \& Security 16, 629-641.

663 Burton, A. M., \& Jenkins, R. (2011). Unfamiliar face perception. In A. J. Calder, G. Rhodes, M. H. 664 Johnson, and J. V. Haxby, editors, Handbook of face perception, pages 287-306, Oxford University $665 \quad$ Press, Oxford, UK.

666 Burton, A. M., Jenkins, R., \& Schweinberger, S. R. (2011). Mental representations of familiar faces. 667 British Journal of Psychology, 102, 943-958.

Burton, A. M., Wilson, S., Cowan, M., \& Bruce, V. (1999). Face recognition in poor quality video: evidence from security surveillance. Psychological Science, 10, 243-248.

Carstens, D. S. (2009). Human and Social Aspects of Password Authentication. In M. Gupta \& R. Shaman (eds.), Social and Human Elements of Information Security: Emerging Trends and Countermeasures, 1-14.

Carstens, D. S., \& Malone, L. C. (2006). Applying Chunking Theory in Organizational Password Guidelines. Journal of Information, Information Technology, and Organizations, 1, 97-113.

Clutterbuck, R., \& Johnston, R. A. (2002). Exploring levels of face familiarity by using an indirect facematching measure. Perception, 31, 985-994.

Clutterbuck, R., \& Johnston, R. A. (2004). Matching as an index of face familiarity. Visual Cognition, 11, 857-869.

Clutterbuck, R., \& Johnston, R. A. (2005). Demonstrating how unfamiliar faces become familiar using a face matching task. European Journal of Cognitive Psychology, 17, 97-116. 
681 682 683 684 685 686 687 688 689 690 691 692 693 694 695 696 697 698 699 700 701 702 703 704 705

Craik, F. I. M., \& Lockhart, R. S. (1972). Levels of processing: A framework for memory research. Journal of Verbal Learning and Verbal behavior, 11, 671-684.

Datta, R., Joshi, D., Li, J., \& Wang, J. Z. (2008). Image Retrieval: Ideas, Influences, and Trends of the New Age. ACM Computing Surveys, 40, 1-60

Davis, D., Monrose, F., \& Reiter, M. (2004). On user choice in graphical password schemes. In $13^{\text {th }}$ USENIX Security Symposium 2004, 151-164.

De Angeli, A., Coventry, L., Johnson, G., \& Renaud, K. (2005). Is a picture really worth a thousand words? On the feasibility of graphical authentication systems. International Journal of Human-Computer Studies, 63, 128-152.

De Haan, E. H. F., Young, A. W., \& Newcombe, F. (1991). A dissociation between sense of familiarity and access to semantic information concerning familiar people. European Journal of Cognitive Psychology, 3, 51-67.

Dhamija, R., \& Perrig, A. (2000). Déjà vu: A user study using images for authentication. In Proceedings of USENIX Security Symposium 2000, 45-58.

Dunphy, P., Nicholson, \& Oliver, P. (2008). Securing Passfaces for Description. In SOUPS 2008. Proceedings of the Fourth Symposium on Usable Privacy and Security, 145, 24-35.

Ebbinghaus, H. (1964). Memory: A Contribution to Experimental Psychology. Trans. H. A. Ruber and C. E. Bussenius. New York: Dover.

Everitt, K. M., Bragin, T., Fogarty, J., \& Kohno, T. (2009). A comprehensive study of frequency, interference, and training of multiple graphical passwords. $C H I$ Proceedings of the 27th international conference on human factors in computing systems 2009, 889898.

Furnell, S., Papadopoulos, I., \& Dowland, P. (2004). A long-term trial of alternative user authentication technologies. Information Management \& Computer Security, 12, $178-$ 190 
706 Garfinkel. S., \& Spafford, G. (1996). Practical UNIX \& Internet security (2nd ed.).

$707 \quad$ Sebastopol, CA: O'Reilly \& Associates.

708 Gehringer, E. F. (2002). Choosing passwords: security and human factors.

709 International Symposium on Technology and Society, 2002, 369-373.

710 Harmon, L. D. (1973). The recognition of faces. Scientific American, 227, 71-82.

711 Jenkins, R., \& Burton, A.M. (2008). 100\% accuracy in automatic face recognition.

$712 \quad$ Science, $319,435$.

713 Jenkins, R., \& Burton, A. M. (2011). Stable face representations. Philosophical

714 Transactions of the Royal Society B, 366, 1671-1683.

715 Jenkins, R., White, D., Montfort, X., \& Burton, A. M. (2011). Variability in photos of the 716 same face. Cognition, 121, 313-323.

717 Lacroix, J. P. W., Postma, E. O., \& Murre, J. M. J. (2005). Predicting experimental

718 similarity ratings and recognition rates for individual natural stimuli with the NIM

719 model. Proceedings of the 27th Annual Meeting of the Cognitive Science Society

720 (Cogsci 2005) (eds. B. Bara, L. Barsalou, and M. Bucciarelli), pp. 1225-1230,

$721 \quad$ Lawrence Erlbaum Associates, Mahwah, NJ.

722 Liu, J., Harris, A., \& Kanwisher, N. (2002). Stages of processing in face perception: An 723 MEG study. Nature Neuroscience, 5, 910-916.

724 Megreya, A. M., \& Burton, A. M. (2006). Unfamiliar faces are not faces: Evidence from 725 a matching task. Memory and Cognition, 34, 865-876.

726 Mihajlov, M., \& Jerman-Blazic, B. (2011). On designing usable and secure recognition727 based graphical authentication mechanisms. Interacting with Computers, 23, 582-593.

728 Miller, G. A. (1956). The magical number seven, plus or minus two: Some limits on 729 our capacity for processing information. Psychological Review 63, 81-97. 
730 Paivio, A., \& Csapo, K. (1973). Picture superiority in free recall: Imagery or dual 731 coding? Cognitive Psychology, 5, 176-206.

732 Podd, J., Bunnell, J., \& Henderson, R. (1996). Cost-effective computer security: 733 Cognitive and associative passwords. In 6th Australian Conference on Computer$734 \quad$ Human Interaction, 1996. 735 736 737

738 739

Sasse, M. A., Brostoff, S., \& Weirich, D. (2001). Transforming the 'weakest link': A human/computer interaction approach to usable and effective security. $B T$ Technology Journal, 19, 122-131.

Sinha, P., Balas, B., Ostrovsky, Y., \& Russell, R., (2006). Face recognition by humans: 19 results all computer vision researchers should know about, Proceedings of the IEEE, 94,1948-1962.

Tam, L., Glassman, M., \& Vandenwauver, M. (2010). The psychology of password management: a tradeoff between security and convenience. Behaviour Information Technology, 29, 233-244

Tari, F., Ozok, A. A., \& Holden, S. H. (2006). A comparison of perceived and real shouldersurfing risks between alphanumeric and graphical passwords. Proceedings of the Second Symposium on Usable Privacy and Security, ACM International Conference Proceedings Series, 149, 56-66.

Valentine, T. (1998). An evaluation of the Passface personal authentication system (Technical Report). London: Goldmsiths College University of London.

Weinshall, D., \& Kirkpatrick, S.(2004). Passwords you'll never forget, but can't recall. In Proceedings of ACM CHI 2004 Conference on Human Factors in Computing Systems, Volume 2 of Late breaking result papers, 1399-1402.

Witty, R. J., \& Brittain, K. (2004). Automated password reset can cut IT service desk costs. Gartner, Inc., Stamford, CT G00123531. 
755 Young, A. W., Hay, D. C., \& Ellis, A. W. (1985). The faces that launched a thousand

756 Slips: Everyday difficulties and errors in recognizing people. British Journal of

757 Psychology, 76: 495-523.

758 Zviran, M., \& Haga, W. J. (1990). Cognitive Passwords: The key to easy access

759 control. Computers and Security, 9, 723-736

Zviran, M., \& Haga, W. J. (1993). A comparison of password techniques for multilevel authentication mechanisms. The Computer Journal, 36, 227-237. 


\section{Figure 1}

Matching a face to a poor quality CCTV image

Figure 1. Example images from Burton, Wilson, Cowan, \& Bruce (1999). Matching poor quality images is easy for observers who are familiar with the faces concerned. Performance of unfamiliar observers is strikingly poor. These images both show the same person.
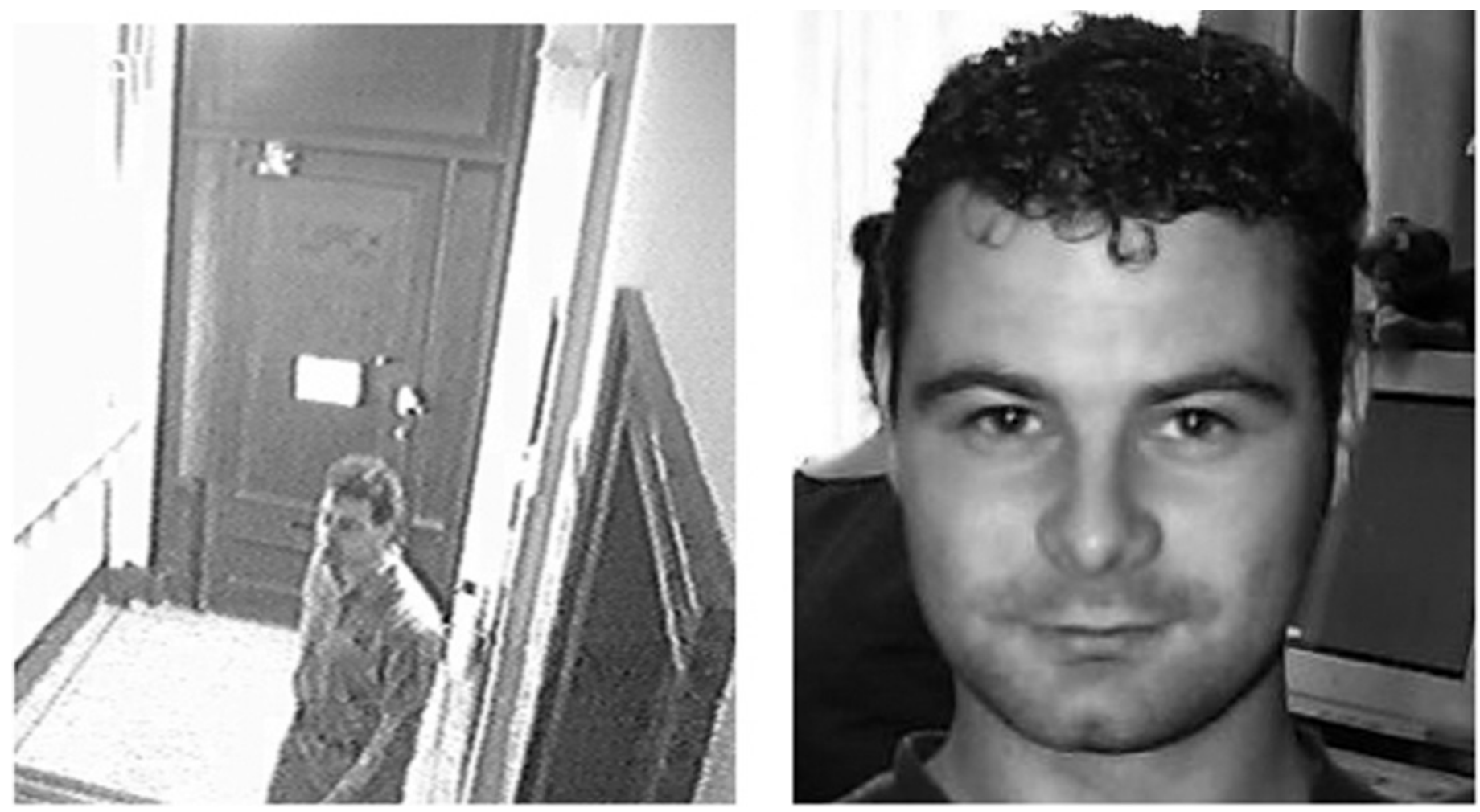


\section{Figure 2}

Familiar and unfamiliar face matching

Figure 2. (a) Matching identical images is trivial. (b) Matching different images of unfamiliar faces is hard. (c) Matching different images of familiar faces is easy. 

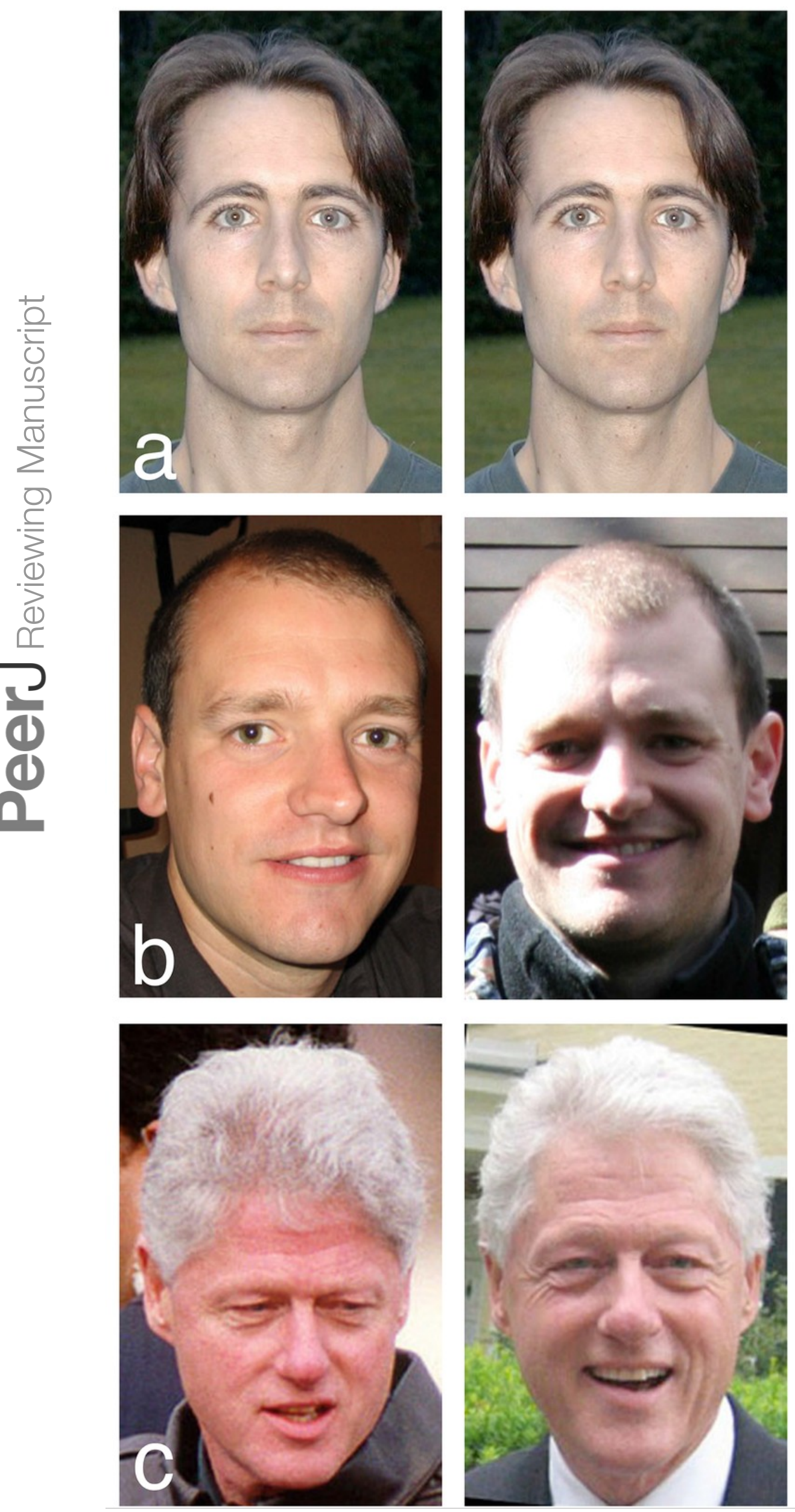

PeerJ reviewing PDF | (v2014:04:1935:1:1:NEW 29 May 2014) 


\section{Figure 3}

Selecting Facelock targets

Figure 3. A schematic diagram summarising the requirements of target faces. If the target is familiar to the attacker, the attacker will be able to authenticate. If the target is unfamiliar to the account holder, the account holder will be unable to authenticate. The tick represents the region of acceptable targets.

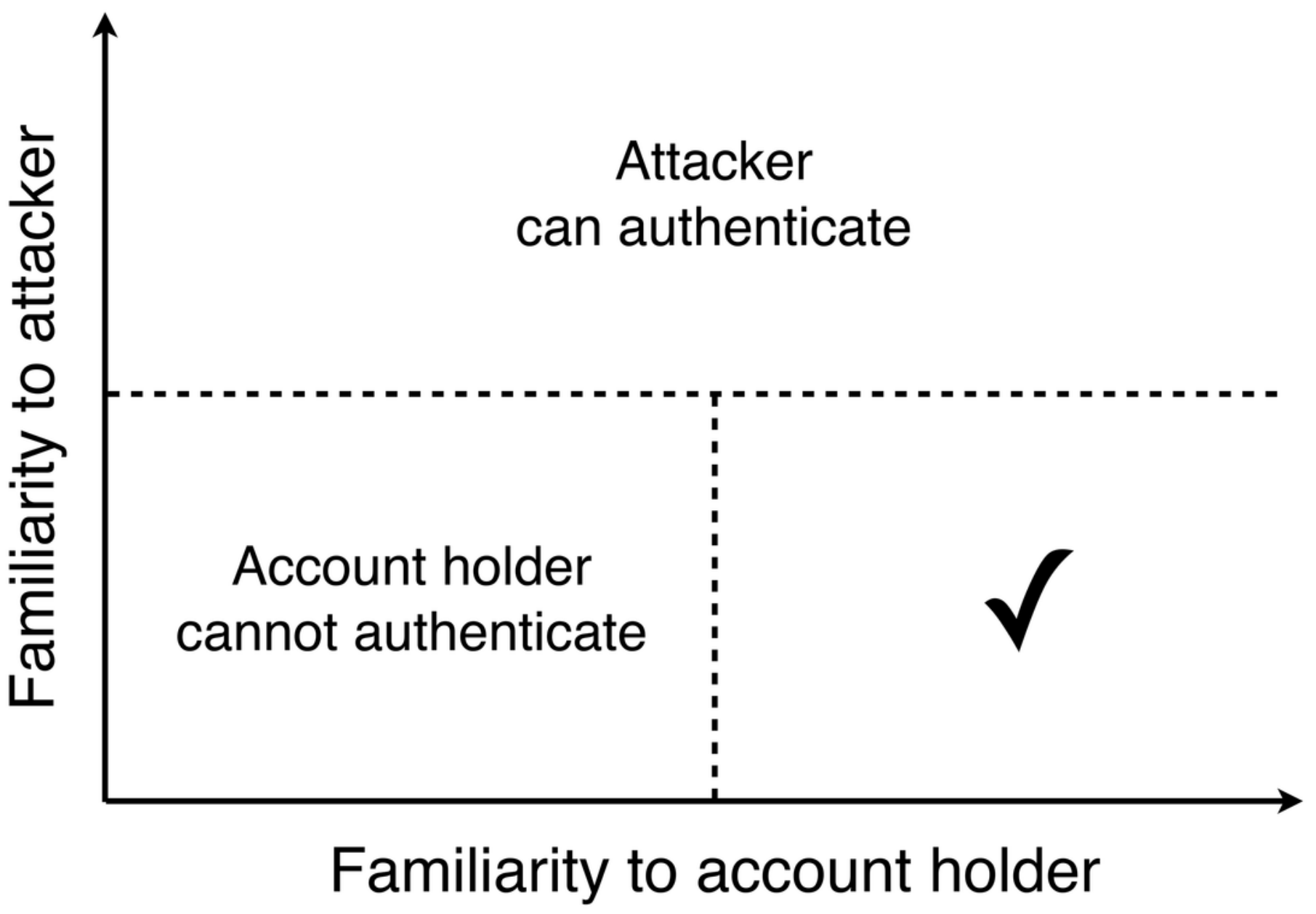




\section{Figure 4}

\section{A challenge grid in Facelock}

Figure 4. An example grid consisting of one face (the target) that is familiar to one of our account holders, and eight faces that are unfamiliar to the same account holder. Readers are invited to guess which of the nine faces is the target. For someone who doesn't know the account holder, it is difficult to find any basis for this decision. 


\section{Figure 5}

Attack success as a function of personal acquaintance

Figure 5. Scatterplot showing the relationship between Personal attackers' Acquaintance Ratings and the number of correctly guessed targets in their first attacks. The area of each datapoint is proportional to the number of cases contributing to it.

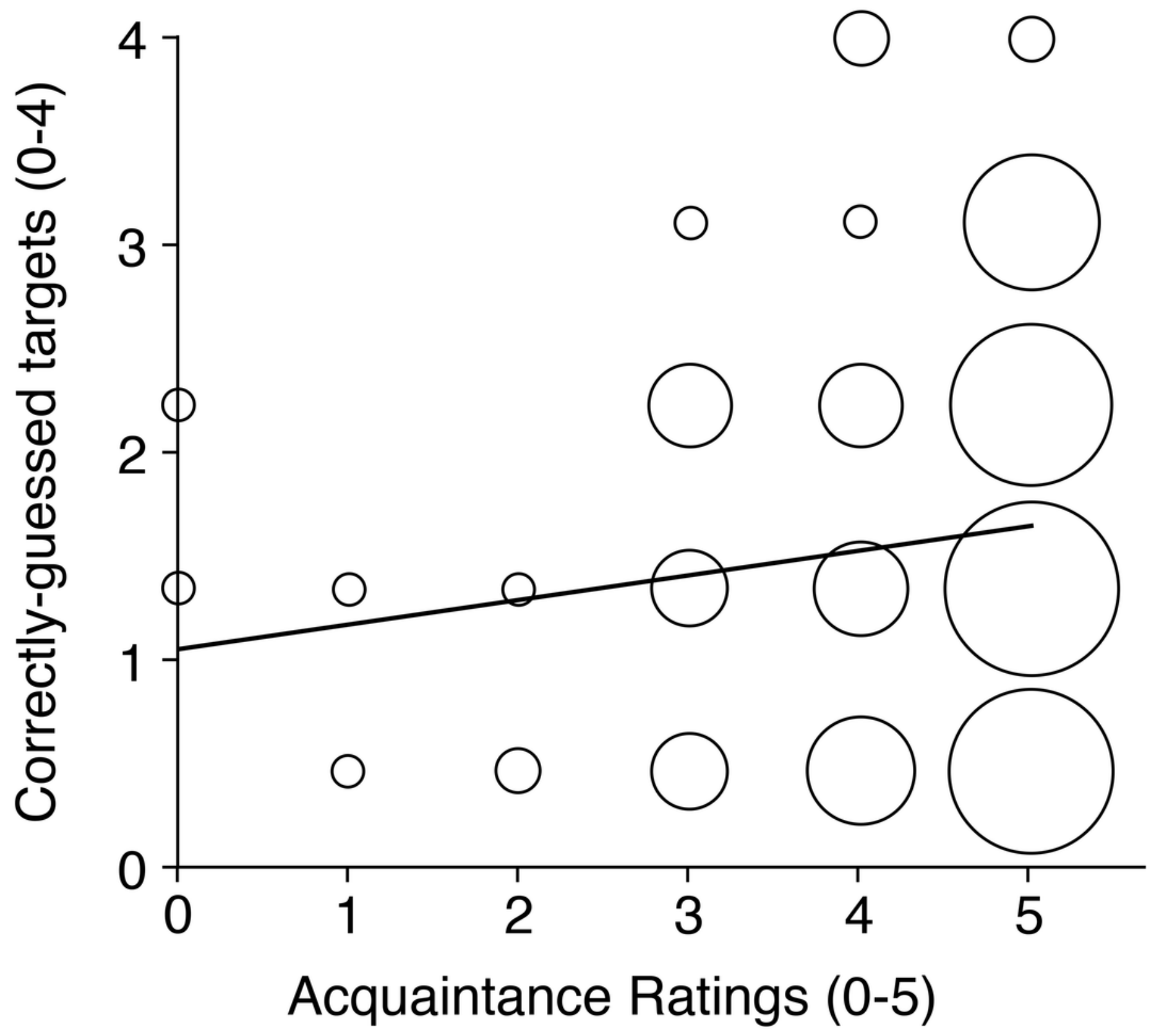




\section{Figure 6}

\section{A second challenge grid}

Figure 6. One of these faces is also present in Figure 4. Even with a single grid, it is difficult to determine which face is repeated simply by trying to memorise Figure 4 . Side-by-side matching of unfamiliar faces is also highly error prone (Jenkins \& Burton, 2008, 2011). 


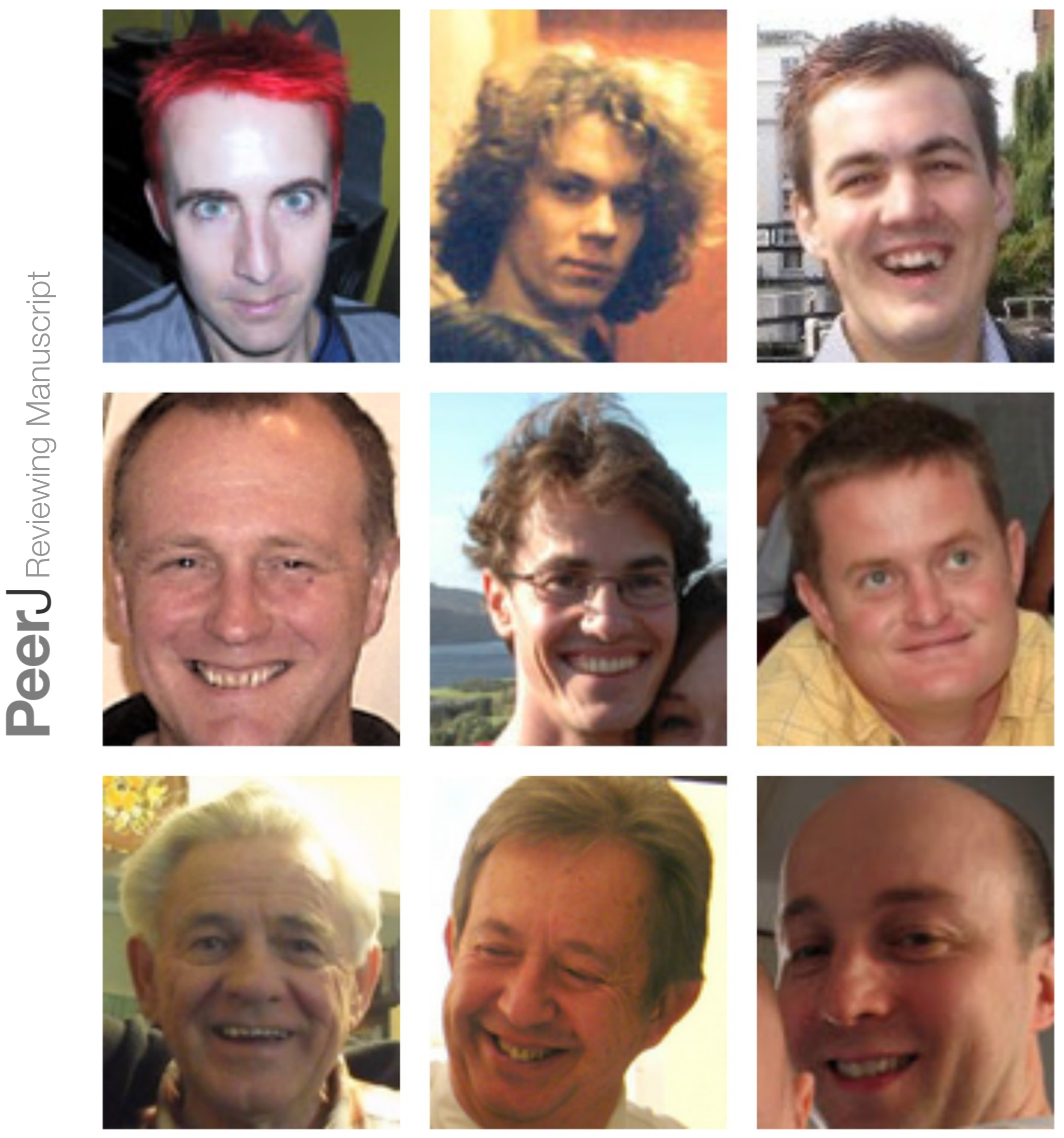




\section{Table 1 (on next page)}

Authentication rates in Study 1.

Table 1. Authentication success rates in Study 1, shown separately for Account Holders and Attackers. See main text for details of delays of procedure. 
Table 1. Authentication success rates in Study 1, shown separately for Account Holders and Attackers. See main text for details of delays of procedure.

\begin{tabular}{|c|c|c|c|c|c|c|c|}
\hline & $N$ & \multicolumn{2}{|c|}{ Succeeded } & Succeeded (1st attempt) & \multicolumn{2}{c|}{ Failed } \\
\hline $\begin{array}{c}\text { Account holders } \\
(1 \text { week delay })\end{array}$ & 120 & 117 & $97.5 \%$ & 101 & $84.1 \%$ & 3 & 11 \\
\hline $\begin{array}{c}\text { Account holders } \\
(1 \text { year delay) }\end{array}$ & 79 & 68 & $86.1 \%$ & 62 & $78.5 \%$ & $13.9 \%$ \\
\hline $\begin{array}{c}\text { Zero-acquaintance } \\
\text { attackers }\end{array}$ & 114 & 1 & $0.9 \%$ & 0 & $0 \%$ & 113 & $99.1 \%$ \\
\hline \begin{tabular}{c} 
Personal attackers \\
\hline
\end{tabular} & 166 & 11 & $6.6 \%$ & 5 & $3.0 \%$ & 155 & $93.4 \%$ \\
\hline
\end{tabular}




\section{Table 2 (on next page)}

Questionnaire data from Study 1.

Table 2. Percentage 'Yes' responses for Account Holders' Questionnaire items from Study 1. 
Table 2. Percentage 'Yes' responses for Account Holders' Questionnaire items from Study 1.

\begin{tabular}{|l|c|}
\hline I wrote my targets' names down to remember them. & $0 \%$ \\
\hline I found it hard to identify my target faces. & $10 \%$ \\
\hline Upon reflection, I would have chosen different target faces. & $80 \%$ \\
\hline I was confused by recognising more than one face in a grid. & $16 \%$ \\
\hline $\begin{array}{l}\text { I would be prepared to use a system like this to log in rather } \\
\text { than a password. }\end{array}$ & $31 \%$ \\
\hline
\end{tabular}




\section{Table 3 (on next page)}

Attacker questionnaire data from Study 1.

Table 3. Mean Likert scale ratings (1-5) for Personal attackers' Questionnaire items from Study 1. 
Table 3. Mean Likert scale ratings (1-5) for Personal attackers' Questionnaire items from Study 1

\begin{tabular}{|l|l|}
\hline How much effort was involved in guessing the targets? & 2.9 \\
\hline $\begin{array}{l}\text { How hard was it to put yourself into the account holder's } \\
\text { shoes to guess his/her targets? }\end{array}$ & 3.5 \\
\hline How successful do you think you were? & 2.3 \\
\hline How well do you know the person? & 4.4 \\
\hline
\end{tabular}




\section{Table 4 (on next page)}

Shoulder-surfing data from Study 2.

Table 4. Shoulder-surfing data from Study 2. Columns refer to the different locks, and rows refer to the number of correctly-guessed targets. All four targets must be correctly guessed for the attacker to gain entry. 
Table 4. Shoulder-surfing data from Study 2. Columns refer to the different locks, and rows refer to the number of correctly-guessed targets. All four targets must be correctly guessed for the attacker to gain entry.

\begin{tabular}{|c|c|c|c|c|c|}
\hline Correctly-guessed targets & Lock 1 & Lock 2 & Lock 3 & Lock 4 & Lock 5 \\
\hline 0 & 9 & 0 & 5 & 15 & 9 \\
\hline 1 & 10 & 4 & 14 & 10 & 13 \\
\hline 2 & 11 & 16 & 12 & 7 & 9 \\
\hline 3 & 2 & 9 & 1 & 0 & 1 \\
\hline 4 & 0 & 3 & 0 & 0 & 0 \\
\hline
\end{tabular}




\section{Table 5 (on next page)}

Threat model.

Table 5. A threat model for Facelock, based on De Angeli et al. (2005). 
Table 5. A threat model for Facelock, based on De Angeli et al. (2005).

\begin{tabular}{|c|c|c|c|}
\hline Threat & Vulnerability & Attack Exploits & Facelock Mitigation \\
\hline Guessability & Predictable choices & Knowledge of a user & Targets are minor celebrities \\
\hline \multirow[t]{2}{*}{ Observability } & Ease of shoulder surfing & Observation of user selecting faces & $\begin{array}{l}\text { Different images of different } \\
\text { targets for each login }\end{array}$ \\
\hline & Ease of intersection attacks & $\begin{array}{l}\text { Refreshing the screen to see } \\
\text { which face stays the same }\end{array}$ & $\begin{array}{l}\text { Different images of different } \\
\text { targets at each refresh } \\
\text { Limited login attempts }\end{array}$ \\
\hline \multirow[t]{2}{*}{ Recordability } & Ease of recording targets' names & User insecure behaviour & $\begin{array}{c}\text { No incentive for account holders } \\
\text { to write down target names }\end{array}$ \\
\hline & Ease of recording the screen & $\begin{array}{l}\text { Use of mobile phone cameras } \\
\text { or screen shots }\end{array}$ & $\begin{array}{l}\text { Different images of different } \\
\text { targets for each login }\end{array}$ \\
\hline
\end{tabular}

